\title{
COMMENTARY
}

\section{Large-scale generation of differentiated cells to achieve regenerative medicine}

\author{
Katsunori Sasaki
}

See related research by Zhang et al., http://stemcellres.com/content/4/6/145

\begin{abstract}
The effects of microgravity and fluid dynamic stress on embryoid bodies generated from pluripotent stem cells induce and direct their differentiation. Using this hydrodynamic effect combined with exogenous factors and three-dimensional culture, a new technique has been developed to produce functional, effective, and safe hepatocytes for transplantation. The evolution of this technique will lead to automated production of a large number of differentiated cells and will significantly contribute to regenerative medicine.
\end{abstract}

To translate regenerative medicine techniques using pluripotent stem cells (PSCs) from laboratories to the clinic, we have to overcome several limitations. The first step is development of simple, inexpensive, and mechanically automated methods to generate large numbers of differentiated cells from undifferentiated PSCs.

Zhang and colleagues proposed a new technique to generate a large number of functional hepatocytes [1]. They developed a three-dimensional culture system using a rotating bioreactor [2]. Hepatic differentiation is induced by microgravity, dexamethasone, dimethyl sulfoxide, hepatocyte growth factor, fibroblast growth factor-4, and insulin-transferrin-selenium at $1 \times 10^{6}$ mouse embryonic stem cells (ESCs) $/ \mathrm{ml}$ and a rotational speed of $25 \mathrm{rpm}$. In the first week of culture, typical embryoid bodies (EBs) appear and then differentiate into hepatocyte-like cells by 3 weeks of culture. This method increases the total number of cells by about $3 \times 10^{3}$-fold. Compared with two-dimensional culture, the generated hepatocyte-like cells express hepatocyte marker genes and produce hepatocyte-associated proteins. Their functions were confirmed by albumin production, the activity

Correspondence: katsmd@shinshu-u.ac.jp

Department of Histology and Embryology, Shinshu University School of Medicine, 3-1-1 Asahi, Matsumoto, Nagano 390-8621, Japan of endogenous cytochrome P450 enzymes, low-density lipoprotein uptake, glycogen production, and indocyanine green staining. Day 14 EB-derived cells were injected into the spleens of nude mice. At 1 month posttransplantation, a large number of EB-derived cells were detected in recipient livers and no tumors were observed in recipient organs. Therefore, hepatocytes grown in this rotating bioreactor are safe and effective for use in transplantation.

The formation of EBs is the first step in conventional methods to differentiate PSCs [3]. EBs consist of the three germ layers and mimic the early period of embryogenesis. Three kinds of research regarding EBs should be taken up to refer to the background, significance, and perspective of the above research.

First, EB size is a parameter that affects differentiation. Bauwens and colleagues created micropatterned Matrigel islands on dishes using a printing method to regulate the size of human ESC colonies (200, 400, and $800 \mu \mathrm{m}$ ) [4]. Small colonies express higher levels of the Gata gene and lower levels of the Pax6 gene, and exhibit endoderm characteristics. Large colonies show converse gene expression and a tendency for neural differentiation. These colonies were collected and resuspended in human ESC differentiation medium to form EBs for 4 days and were then transferred to plates for EB outgrowth. Among EBs generated from a high ratio of Gata/Pax6-positive colonies, larger EBs showed higher mesoderm and cardiac induction. Conversely, among EBs generated from a low ratio of Gata6/Pax6-positive colonies, smaller EBs exhibited higher cardiac induction. These results suggest that differentiation may be controlled by changing the EB size.

Second, the speed of the rotary culture regulates EB size. Carpenedo and colleagues demonstrated that rotary motion at $25 \mathrm{rpm}$ generates the largest and fewest EBs from mouse ESCs, and $55 \mathrm{rpm}$ yields the smallest EBs [5]. These generated EBs were uniform and expressed marker genes of the three germ layers. The EB size can 
therefore be controlled without the micropatterning technique. Moreover, an increase of endodermal gene expression and histological examination of cystic EB formation suggest that differentiation toward endoderm is accelerated in rotary-formed EBs.

Third, rotary culture involves fluid dynamics and microgravity that regulate EB differentiation. For example, ESCs exposed to fluid shear stress $\left(5 \mathrm{dyn} / \mathrm{cm}^{2}\right)$ show increased expression of endothelial marker genes, and EBs generated from these cells demonstrate the same tendency [6]. Rotary culture not only forms EBs and regulates their size, but also causes varied fluid dynamics and microgravity, and applies shear stress and gravitational effects on PSCs and EBs. Changing the fluid dynamics and microgravity may result in more selective differentiation.

Computational analysis combined with rotary culture by Sargent and colleagues revealed that different fluid dynamics occur in rotating dishes at 25,40 , and $55 \mathrm{rpm}$ [7]. This finding suggests that shear stress modulates the EB structure including the cellular organization and morphology of the spheroids. Compared with static culture, rotary culture increases the expression of cardiomyocyte marker genes. Mogi and colleagues have shown that $80 \%$ of EBs created at rapid speed (100 rpm) beat after outgrowth without the addition of growth factors [8]. However, at $120 \mathrm{rpm}$ the number of beating EBs decreases by one-half. Computational fluid dynamic analysis showed abrupt changes of the fluid dynamic phase from 100 to $120 \mathrm{rpm}$. Zhang and colleagues found that selective differentiation by microgravity is able to generate cells with endodermal phenotypes from PSCs [1].

Microgravity-associated methods may realize selective differentiation of PSCs to supply large quantities of differentiated cells and satisfy the demand of regenerative medicine. These methods may not be fully developed, suitable equipment has not been established, and the optimal conditions have not been determined. However, such limitations may be overcome by investigating techniques such as three-dimensional culture systems and the addition of exogenous factors as suggested by Zhang and colleagues [1].

\section{Abbreviations}

EB: Embryoid body; ESC: Embryonic stem cell; PSC: Pluripotent stem cell.

\section{Competing interests}

The author declares that he has no competing interests.

Published: 20 Jan 2014

\section{References}

1. Zhang S, Zhang Y, Chen L, Liu T, Li Y, Wang Y, Geng Y: Efficient large-scale generation of functional hepatocytes from mouse embryonic stem cells grown in a rotating bioreactor with exogenous growth factors and hormones. Stem Cell Res Ther 2013, 4:145.
2. Wang $Y$, Zhang $Y$, Zhang S, Peng G, Liu T, Li Y, Xiang D, Wassler MJ, Shelat HS, Geng Y: Rotating microgravity-bioreactor cultivation enhances the hepatic differentiation of mouse embryonic stem cells on biodegradable polymer scaffolds. Tissue Eng Part A 2012, 18:2376-2385.

3. Itskovitz-Eldor J, Schuldiner M, Karsenti D, Eden A, Yanuka O, Amit M, Soreq $\mathrm{H}$, Benvenisty N: Differentiation of human embryonic stem cells into embryoid bodies compromising the three embryonic germ layers. $\mathrm{Mol}$ Med 2000, 6:88-95.

4. Bauwens CL, Peerani R, Niebruegge S, Woodhouse KA, Kumacheva E, Husain $M$, Zandstra PW: Control of human embryonic stem cell colony and aggregate size heterogeneity influences differentiation trajectories. Stem Cells 2008, 26:2300-2310.

5. Carpenedo RL, Sargent CY, McDevitt TC: Rotary suspension culture enhances the efficiency, yield, and homogeneity of embryoid body differentiation. Stem Cells 2007, 25:2224-2234.

6. Nsiah BA, Ahsan T, Griffiths S, Cooke M, Nerem RM, McDevitt TC: Fluid shear stress pre-conditioning promotes endothelial morphogenesis of embryonic stem cells within embryoid bodies. Tissue Eng Part A 2013 [Epub ahead of print].

7. Sargent CY, Berguig GY, Kinney MA, Hiatt LA, Carpenedo RL, Berson RE, McDevitt TC: Hydrodynamic modulation of embryonic stem cell differentiation by rotary orbital suspension culture. Biotechnol Bioeng 2010, 105:611-626.

8. Mogi A, Takei S, Shimizu H, Miura H, Tomotsune D, Sasaki K: Effects of fluid dynamic forces created by rotary orbital suspension culture on cardiomyogenic differentiation of human embryonic stem cells. J Med Biol Eng 2013: [Epub ahead of print; doi:10.5405/jmbe.1356].

$10.1186 /$ scrt399

Cite this article as: Sasaki: Large-scale generation of differentiated cells to achieve regenerative medicine. Stem Cell Research \& Therapy 2014, 5:10 